\title{
MULTIPLE VALUED ITERATIVE DYNAMICS MODELS OF NONLINEAR DISCRETE-TIME CONTROL DYNAMICAL SYSTEMS WITH DISTURBANCE
}

\author{
BYUNGIK KAHNG
}

\begin{abstract}
The study of nonlinear discrete-time control dynamical systems with disturbance is an important topic in control theory. In this paper, we concentrate our efforts to multiple valued iterative dynamical systems, which model the nonlinear discrete-time control dynamical systems with disturbance. After establishing the validity of such modeling, we study the invariant set theory of the multiple valued iterative dynamical systems, including the controllability/reachablity problems of the maximal invariant sets.
\end{abstract}

\section{Introduction}

The importance of the invariant set theory in control and automation theory is well documented. See, for instance, $[5,15,16]$ and the references therein for the applications of the invariant set theory in classical nonlinear discrete-time control dynamical systems. The invariant set theory of nonlinear discrete-time control dynamical systems with disturbance, which we abbreviate as disturbed control dynamical system $(D C D S)$ for the remainder of this paper, is relatively new, at least in the realm of pure mathematics. In engineering, however, there is already a substantial amount of literature devoted to this topic, such as $[3,20,21,22,23]$, to name a few.

The presence of the disturbance makes the DCDS more realistic and useful, but at the same time, more difficult. In contrast to the classical undisturbed counterpart, a DCDS cannot be reduced to an iterative dynamical system of one endomorphism, or more informally, a closed-loop system. Hence, when studying the DCDS, we are often forced to deal with the difficulties associated with the open-loop systems. The purpose of multiple valued iterative dynamics modeling (MVID modeling) is to overcome such difficulties by closing-up the

Received August 24, 2011; Revised June 23, 2012.

2010 Mathematics Subject Classification. Primary 37N35, 93B05, 93C10, 93C30, 93C55; Secondary 93A30, 93B03, 93B52, 93C85.

Key words and phrases. nonlinear discrete-time control dynamical system, disturbance, invariance, steady state, controllability/reachability. 
open-loops so that the results analogous to those of undisturbed control dynamical systems can be established. The MVID modeling is based upon the theory of multiple valued iterative dynamical systems (MVIDS). It is similar to the method of set valued functions that was used successfully by [3] and [22] in some reachability/controllability problems and their approximation problems.

The purpose of this paper is to establish the MVID modeling of DCDS and investigate the invariant set theory of the MVIDS. The MVID modeling of DCDS was introduced in [9], in somewhat primitive form. It was subsequently improved by [10]. This paper improves it further through more rigorous treatment (Section 2). The invariant set theory of the MVIDS was studied in [9] and [10], too. However, the main contributions of [9] and [10] were confined to the easier half of the invariant set theory of MVIDS, namely quasi-invariance

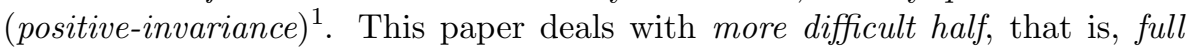
invariance (Section 4 and Section 5). The full invariance is intimately related to the steady state of the dynamics, or the long-term behavior of the orbits. Also, a concrete, albeit elementary, numerical example will be discussed in the appendix (Section 6).

This article is structured as follows. In Section 2, we review the typical models of DCDS, and then, rigorously construct the MVID model (Proposition 2.2). The comparison between the traditional models of DCDS and the MVID model was, for the most part, done in [10], but it will be re-examined here for the sake of the self-sufficiency (Proposition 2.3). After a brief review on the invariant set theory of the single valued iterative dynamical system in Section 3 , we define and characterize various concepts of invariance for MVIDS in Section 4 (Definition 4.6 and Theorem 4.9). We use the concepts of orbitchains (Definition 4.1) and predecessor operators (Definition 4.4) as the main tools. The reachability/controllability problems of maximal invariant sets follow in Section 5. Finally, in Section 6, a simple MVIDS-specific example of the infinite-step controllability and finite-step approximate control problem will be presented as an appendix.

\section{Modeling disturbed control dynamical systems}

\subsection{Classical models of nonlinear discrete-time control dynamical systems with deterministic disturbance}

It is well known that the classical non-linear time-invariant discrete-time deterministic control dynamical system given by the pair of maps $f: X \times U \rightarrow$

\footnotetext{
${ }^{1}$ The strong and weak quasi-invariance of MVIDS and the reachability/controllability problems of the maximal quasi-invariant sets were discussed in the author's earlier contribution, [10]. The brief review of these topics we present here do not contain the technical proofs.
} 
$X$ and $g: X \rightarrow U$, where

$$
\left\{\begin{array}{l}
f:\left(x_{k}, u_{k}\right) \mapsto x_{k+1}, \\
g: x_{k} \mapsto u_{k},
\end{array}\right.
$$

can be reduced the iterative dynamics of one endomorphism $\psi: X \rightarrow X$ where

$$
\psi: x \mapsto f(x, g(x))
$$

Such a reduction is valid because of the time-invariance. The input variable $u_{k} \in U$ depends only on the current state $x_{k} \in X$, so the feedback control law $g: x_{k} \rightarrow u_{k}$ is a well-defined single valued map. Putting $f$ and $g$ together, therefore, we get the iterative dynamical system (2.2) of one endomorphism, $\psi$. This reduction and the invariant set theory of the iterative dynamical system (2.2) are important tools in the classical applications of control and automation theory such as model predictive control $[6,16,17,18]$.

These days, the dynamics of the systems (2.1) and (2.2) are attracting renewed attention, as evidenced by the large amount of modern literature on this topic. See, for instance, $[3,15,20,21,23]$ and the references therein. The focus of more recent research, however, revolves around the time-dependent disturbance. In general, the disturbance does not apply to a given state the same way at any given time, or it is not a disturbance at all. Therefore, neither $f$ nor $g$ is well defined (single valued) function, under the presence of the disturbance.

One way to express the disturbance is to perturb the dynamics $f$ and the feedback control law $g$ of the system (2.1), by inserting some extra variables $v_{k} \in V$ and $w_{k} \in W$, which are often called disturbance variables. That is,

$$
\left\{\begin{array}{l}
f_{w}:\left(x_{k}, u_{k}, w_{k}\right) \mapsto x_{k+1}, \\
g_{v}:\left(x_{k}, v_{k}\right) \mapsto u_{k} .
\end{array}\right.
$$

Here, the control dynamics model (2.3) is slightly more general version than the traditional method to model the DCDS, in a sense that the feedback control map $g$ is also perturbed. Compare the system (2.3) with those considered in $[15,23]$, for instance.

The DCDS given by (2.3) can be reduced to the iterative dynamics of $\psi$ : $X \times V \times W \rightarrow X \times V \times W$, where

$$
\psi:(x, v, w) \mapsto(f(x, g(x, v), w), \phi(v, w)),
$$

and $\phi: V \times W \rightarrow V \times W$. The obvious advantage of this reduction is the simplicity. However, this reduction is problematic in the following sense. The map $\phi$ is, and should be, unknown, and thus, so is $\psi$. In other words, we cannot predict which disturbance would take place and how it would affect our system, or it would not be a disturbance at all. 
Another well known method is to make the system stochastic, by making all the variables time-dependent, as in [20], among others. That is,

$$
\left\{\begin{array}{l}
f_{t}:(x(t), u(t), t) \mapsto x(t+1), \\
g_{t}:(x(t), t) \mapsto u(t) .
\end{array}\right.
$$

This model can also be reduced to an iterative dynamics, through

$$
\psi:(x, t) \mapsto(f(x, g(x, t), t), t+1),
$$

where $\psi: X \times T \rightarrow X \times T$ combines the dynamics of $f$ and $g$ of the system (2.4). This time, the second component $t \mapsto t+1$ can be understood as the natural elapse of time by one time unit.

By taking $t \in \mathbb{R}$, we get a continuous-time dynamical system, or a flow in the phase space [2]. Upon generalizing the second component $t \mapsto t+1$ to $t_{k} \mapsto t_{k}+\Delta_{k} t$, we get a variable-time-scale dynamical system [1], an interesting mathematical topic on its own. In the viewpoint of control and automation theory, however, there is a problem about the second approach, too. How the disturbance at a given time affects the dynamics is, and should be, difficult to predict. Consequently, the same problem that affected system (2.3) follows.

\subsection{Multiple valued iterative dynamics (MVID) models of disturbed control dynamical systems (DCDS)}

In this paper, we use an alternative approach to model the DCDS. We will allow $f$ and $g$ in (2.1) to be multiple valued. Consequently, we get an iterative dynamical system given by a multiple valued map $\psi(x)=f(x, g(x))$. Let us elaborate this idea as follows.

Definition 2.1 (Multiple Valued Map and Multiple Valued Iterative Dynamics). Let $X, Y$ be non-empty sets, and $\mathscr{P}(X), \mathscr{P}(Y)$ be their power sets. We say a set function $\psi: \mathscr{P}(X) \rightarrow \mathscr{P}(Y)$ is a multiple valued map (function) from $X$ to $Y$ if

$$
\psi(S)=\bigcup\{\psi(x): x \in S\}
$$

for all $S \subset X$. Here, $\psi(x)$ is the abbreviation of $\psi(\{x\})$. Let us denote the set of all multiple valued maps from $X$ to $Y$, as $\mathscr{M}\left(Y^{X}\right)$. In particular, if $X=Y$, we call the iterative dynamical system in $X$ given by the multiple valued self-map $\psi \in \mathscr{M}\left(X^{X}\right)$, the multiple valued iterative dynamical system $(M V I D S)$.

For the remainder of this paper, we will regard $\mathscr{P}(\cdot)$ and $\mathscr{M}(\cdot)$ denote the power set and the class of multiple valued maps as in Definition 2.1. In contrast to the MVIDS, a classical iterative dynamical system of a single valued self-map will be referred as a single valued iterative dynamical system (SVIDS), when it becomes necessary to distinguish the two.

The first step toward the main results of this paper is to establish the following: (1) how any DCDS can be modeled by a MVIDS, and (2) why the MVIDS 
turns out to be the most general model yet. The following propositions answer these questions.

Proposition 2.2 (The Validity of MVID Modeling). Let X be a nonempty set, which we will call the phase space, and whose elements we will call the states. Then, any DCDS can be modeled as an MVIDS in a such a way that each iterate of the MVIDS consists precisely of all possible outcomes that we must account for. Let us call this process a multiple valued iterative dynamics modeling (MVID modeling) of the given DCDS.

Proposition 2.3 (The Generality of MVID Modeling [10]). Let $U, V, W$ and $X$ be nonempty sets, and let $\mathscr{P}(X)$ be the power set of $X$. Also, let $f: X \times U \times W \rightarrow X$ and $g: X \times V \rightarrow U$ be maps. Then the set function $\psi: \mathscr{P}(X) \rightarrow \mathscr{P}(X)$ given by

$$
\psi(A)=\{f(x, g(x, v), w): v \in V, w \in W, x \in A\}
$$

satisfies the condition (2.5), and thus, $\psi \in \mathscr{M}\left(X^{X}\right)$.

Also, if $T, U$ and $X$ are nonempty sets, the set function $\psi: \mathscr{P}(X) \rightarrow \mathscr{P}(X)$ given by

$$
\psi(A)=\{f(x, g(x, t), t): t \in T, x \in A\}
$$

satisfies the condition (2.5) as well, consequently yielding $\psi \in \mathscr{M}\left(X^{X}\right)$.

The proof of Proposition 2.3 can be found in [10]. About Proposition 2.2, let us paraphrase it and elaborate it through the following observations. They are rather trivial in terms of depth, but their implication is significant in that they validate the very model that this paper is based upon.

Further Comments about Proposition 2.2. Given initial state $x \in X$, let $\psi(x)$ $\subset X$ be the set of all possible outcomes of the DCDS that originate from $x$. Now, define a set function $\psi: \mathscr{P}(X) \rightarrow \mathscr{P}(X)$ by the equality (2.5). Because the set function $\psi$ was defined through the equality $(2.5), \psi \in \mathscr{M}\left(X^{X}\right)$ follows immediately from Definition 2.1.

Repeating the same process to each element of $\psi(x)$, we get the second iteration of the DCDS. Continuing this process, we get a MVIDS in $X$ given by $\psi \in \mathscr{M}\left(X^{X}\right)$, which models the given discrete-time dynamical system with deterministic disturbance. Furthermore, from the construction of $\psi$, it is clear that each $\psi^{k}(x)$ consists of all possible outcomes that originated from $x$ in $k$-th step, and contains nothing else.

Let us call the MVIDS given by the iteration of $\psi \in \mathscr{M}\left(X^{X}\right)$, the multiple valued iterative dynamics model (MVID model) of the given DCDS.

\section{Invariant set theory of single valued iterative dynamical systems}

\subsection{Invariance and maximal invariance}

In, this section, we review the invariance concept of SVIDS. 
Definition 3.1 (Invariance and Quasi-invariance). Let $X$ be a non-empty set, and let $\psi: X \rightarrow X$. A set $S \subset X$ is said to be invariant under $\psi$ if $\psi(S)=S$. Also, $S$ is said to be quasi-invariant, if $\psi(S) \subset S$, or equivalently, $S \subset \psi^{-1}(S)$.

Proposition 3.2 (Quasi-invariance and Positive Invariance). Let $X$ be a nonempty set, and let $\psi: X \rightarrow X$. Then, a set $S \subset X$ is quasi-invariant if and only if it is positive-invariant. That is, $\psi(S) \subset S$ if and only if $x_{0} \in S$ implies that $x_{k}=\psi^{k}(x) \in S$ for every $k \in \mathbb{Z}^{+}$.

The proof of Proposition 3.2 is trivially simple, so we leave it to the readers. Instead, we state and prove a stronger theorem in Subsection 4.3 (Theorem 4.9).

Definition 3.3 (Maximal Invariant Sets of SVIDS). Let $X$ and $Y$ be nonempty sets where $Y \subset X$, and let $\psi: X \rightarrow X$. We define the locally maximal invariant subset $\mathcal{M}(Y)$ of $Y$, and the locally maximal quasi-invariant subset $\mathcal{M}^{+}(Y)$ of $Y$ by,

$$
\left\{\begin{array}{l}
\mathcal{M}(Y)=\bigcup\{S \subset Y: \psi(S)=S\} \\
\mathcal{M}^{+}(Y)=\bigcup\{S \subset Y: \psi(S) \subset S\}
\end{array}\right.
$$

In the special case, $Y=X$, we call $\mathcal{M}(X)$, the (globally) maximal invariant set. When there is no danger of confusion, we will use the plural term, maximal invariant sets to refer to all three at the same time.

The proof of the maximality, invariance and quasi-invariance are easy and left to the readers. Instead, we prove a stronger result in Subsection 5.1 (Lemma $5.2)$.

\subsection{Controllability problems of maximal invariant sets}

Definition 3.3 ensures the existence of the maximal invariant sets, but it does not help us determining them algorithmically. Under some suitable conditions, it is known that the maximal invariant sets are $\infty$-step controllable $[10,14]$. Hence, the finite-step approximate control problems and their optimization problems are well-posed. Moreover, the approximation can be done through the following iterative algorithms. See $[10,14]$ for more detail.

Proposition 3.4 (Controllability of Locally Maximal Quasi-invariant Sets). Let $X$ and $Y$ be nonempty sets, $Y \subset X$, and $\psi: X \rightarrow X$. Suppose further that $Y^{0}=Y$ and $Y^{-k}=Y \cap \psi^{-1}\left(Y^{-(k-1)}\right)$. Then,

$$
Y^{0} \supset Y^{-1} \supset Y^{-2} \supset \cdots \supset \bigcap_{k=0}^{\infty} Y^{-k}=\mathcal{M}^{+}(Y) .
$$

Theorem 3.5 (Controllability of Locally Maximal Full-invariant Sets $[10,14]$ ) Let $X$ be a non-empty topological space and $\psi: X \rightarrow X$. Let $Y$ be a nonempty subspace of $X$. Suppose further that $Y^{0}=Y, Y^{k}=Y \cap \psi\left(Y^{k-1}\right)$, and $Y^{-k}=$ $Y \cap \psi^{-1}\left(Y^{-(k-1)}\right)$. Finally, suppose that one of the following two conditions 
hold: either (1) $Y$ is compact and $\left.\psi\right|_{Y}: Y \rightarrow X$ is continuous, or (2) $\left.\psi\right|_{Y}$ : $Y \rightarrow X$ is finite-to-one. Then,

$$
Y^{0} \supset\left(Y^{1} \cap Y^{-1}\right) \supset\left(Y^{2} \cap Y^{-2}\right) \supset \cdots \supset \bigcap_{k=0}^{\infty}\left(Y^{k} \cap Y^{-k}\right)=\mathcal{M}(Y) .
$$

\section{Multiple valued iterative dynamical systems}

\subsection{Orbit-chains}

We begin discussing MVIDS with its most important aspect, the orbitbehavior. The orbits of SVIDS are given by the iterative dynamics of individual states. The corresponding quantities of MVIDS, however, are not so simple.

Definition 4.1 (Orbit-chains). Let $X$ be a nonempty set and $\psi \in \mathscr{M}\left(X^{X}\right)$. Let $Y \subset X$ and $x_{0} \in Y$.

A. Let $n \in \mathbb{Z}^{+}$. We call a finite sequence $\left(x_{k}\right)_{k=0}^{n}$ such that $x_{k} \in \psi\left(x_{k-1}\right) \cap Y$ for each $k \in\{1, \ldots, n\}$, a forward orbit-chain of $x_{0}$ of length $n$ in $Y$, and write $\left(x_{k}\right)_{k=0}^{n} \in \mathscr{O}^{n}\left(x_{0}: Y\right)$. If $\mathscr{O}^{n}\left(x_{0}: Y\right)=\mathscr{O}^{n}\left(x_{0}: X\right)$, then we denote $x_{0} \in \mathcal{F}_{s}^{n}(Y)$. If $\mathscr{O}^{n}\left(x_{0}: Y\right) \neq \emptyset$, then we say $x_{0}$ admits a forward orbit-chain of $x_{0}$ of length $n$ in $Y$, and denote $x_{0} \in \mathcal{F}_{w}^{n}(Y)$.

B. We call an infinite sequence $\left(x_{k}\right)_{k=0}^{\infty}$ such that $x_{k} \in \psi\left(x_{k-1}\right) \cap Y$ for each $k \in$ $\mathbb{Z}^{+}$, an infinite forward orbit-chain of $x_{0}$ in $Y$, and write $\left(x_{k}\right)_{k=0}^{n} \in \mathscr{O}^{+}\left(x_{0}: Y\right)$. If $\mathscr{O}^{+}\left(x_{0}: Y\right)=\mathscr{O}^{+}\left(x_{0}: X\right)$, then we write $x_{0} \in \mathcal{F}_{s}^{\omega}(Y)$. If $\mathscr{O}^{+}\left(x_{0}: Y\right) \neq \emptyset$, then we say $x_{0}$ admits an infinite forward orbit-chain of $x_{0}$ in $Y$, and denote $x_{0} \in \mathcal{F}_{\omega}(Y)$. Finally, if $\mathscr{O}^{n}\left(x_{0}: Y\right) \neq \emptyset$ for every $n \in \mathbb{Z}^{+}$, then we denote $x_{0} \in \mathcal{F}_{w}^{\infty}(Y)$.

C. Let $n \in \mathbb{Z}^{+}$. We call a finite sequence $\left(x_{-k}\right)_{k=0}^{n}$ such that $x_{-(k-1)} \in$ $\psi\left(x_{-k}\right) \cap Y$ for each $k \in\{1, \ldots, n\}$, a backward orbit-chain of $x_{0}$ of length $n$ in $Y$, and write $\left(x_{-k}\right)_{k=0}^{n} \in \mathscr{O}^{-n}\left(x_{0}: Y\right)$. If $\mathscr{O}^{-n}\left(x_{0}: Y\right) \neq \emptyset$, then we say $x_{0}$ admits a backward orbit-chain of $x_{0}$ of length $n$ in $Y$, and denote $x_{0} \in \mathcal{B}^{n}(Y)$.

D. We call an infinite sequence $\left(x_{-k}\right)_{k=0}^{\infty}$ such that $x_{-(k-1)} \in \psi\left(x_{-k}\right) \cap Y$ for each $k \in \mathbb{Z}^{+}$, an infinite backward orbit-chain of $x_{0}$ in $Y$, and write $\left(x_{-k}\right)_{k=0}^{n} \in$ $\mathscr{O}^{-}\left(x_{0}: Y\right)$. If $\mathscr{O}^{-}\left(x_{0}: Y\right) \neq \emptyset$, then we say $x_{0}$ admits an infinite backward orbit-chain of $x_{0}$ in $Y$, and denote $x_{0} \in \mathcal{B}^{\omega}(Y)$. Finally, if $x_{0}$ admits a backward orbit-chain of every finite length in $Y$, that is, $\mathscr{O}^{-n}\left(x_{0}: Y\right) \neq \emptyset$ for every $n \in \mathbb{Z}^{+}$, then let us write $x_{0} \in \mathcal{B}^{\infty}(Y)$.

Needless to say, we get the following immediate result.

Proposition 4.2. Let $X$ be a nonempty set and $\psi \in \mathscr{M}\left(X^{X}\right)$. Then for any $Z \subset Y \subset X$, we must have $\mathcal{F}_{s}^{\nu}(Z) \subset \mathcal{F}_{s}^{\nu}(Y), \mathcal{F}_{w}^{\nu}(Z) \subset \mathcal{F}_{w}^{\nu}(Y)$, and $\mathcal{B}^{\nu}(Z) \subset \mathcal{B}^{\nu}(Y)$, where $\nu=n \in \mathbb{Z}^{+}, \nu=\infty$, or $\nu=\omega$.

In trivial case $(n=0)$, let us write $\left(x_{0}\right) \in \mathscr{O}^{0}(Y)$ and $\mathcal{F}_{s}^{0}(Y)=\mathcal{F}_{w}^{0}(Y)=$ $\mathcal{B}^{0}(Y)=Y$. Also, we can tentatively write $x_{0} \in \mathcal{F}_{s}^{\infty}(Y)$ if $\mathscr{O}^{n}\left(x_{0}: Y\right)=$ 
$\mathscr{O}^{n}\left(x_{0}: X\right)$ for every $n \in \mathbb{Z}^{+}$. Under these additional trivialities, we get the following result.

Lemma 4.3 (Descending Chain Lemma). Let $X$ be a nonempty set and $\psi \in$ $\mathscr{M}\left(X^{X}\right)$. Then for any $Y \subset X$, we get

$$
\begin{aligned}
& \mathcal{F}_{s}^{0}(Y) \supset \mathcal{F}_{s}^{1}(Y) \supset \cdots \supset \mathcal{F}_{s}^{\infty}(Y)=\mathcal{F}_{s}^{\omega}(Y), \\
& \mathcal{F}_{w}^{0}(Y) \supset \mathcal{F}_{w}^{1}(Y) \supset \cdots \supset \mathcal{F}_{w}^{\infty}(Y) \supset \mathcal{F}_{w}^{\omega}(Y), \\
& \mathcal{B}^{0}(Y) \supset \mathcal{B}^{1}(Y) \supset \cdots \supset B^{\infty}(Y) \supset B^{\omega}(Y) .
\end{aligned}
$$

Sketch of Proof. The descending chain-relations are obvious from the definitions. Only the lone equality deserves our attention. Let $x_{0} \in \mathcal{F}_{s}^{\infty}(Y)$ and choose any $\left(x_{k}\right)_{k=0}^{\infty} \in \mathscr{O}^{+}\left(x_{0}: X\right)$. Then, for each $n \in \mathbb{Z}^{+},\left(x_{k}\right)_{k=0}^{n} \in \mathscr{O}^{n}\left(x_{0}\right.$ : $X)=\mathscr{O}^{n}\left(x_{0}: Y\right)$, and thus, $x_{n} \in Y$. Hence, $x_{0} \in \mathcal{F}_{s}^{\omega}(Y)$. This proves, $\mathcal{F}_{s}^{\infty}(Y) \subset \mathcal{F}_{s}^{\omega}(Y)$, which then leads to the equality.

In the viewpoint of the MVID modeling of DCDS, we can regard each orbitchain in $X$ as one possible chain of events up to $n$ iterations of the dynamics. Each orbit-chain in $Y$, on the other hand, can be regarded as one possible chain of events that takes place entirely in $Y$. In other words, the concept of the orbit-chains connects the set-dynamics given by MVIDS and the chain of actual events of the DCDS expressed by MVID modeling.

\subsection{Pre-image sets and predecessor operators}

For the classical theory of SVIDS, the predecessor operator $[5,15,16,23,24]$ of a single valued iterative dynamical system given by the endomorphism $\psi$ : $X \rightarrow X$ is nothing more than the pre-image map, $\psi^{-1}: \mathscr{P}(X) \rightarrow \mathscr{P}(X)$, $\psi^{-1} \in \mathscr{M}\left(X^{X}\right)$. The pre-images and predecessors of MVIDS, however, are not so straightforward, as we will see in the following definition.

Definition 4.4 (Pre-images and Predecessors). Let $X$ be a nonempty set and $\psi \in \mathscr{M}\left(X^{X}\right)$. Given nonempty subset $S \subset X$, we define the strong pre-image set of $S$ as,

$$
\psi_{s}^{-1}(S)=\{x \in X: \psi(x) \subset S\} .
$$

In general, we define the $k$-th strong pre-image set of $S$ by

$$
\psi_{s}^{-k}(S)=\left\{x \in X: \psi^{k}(x) \subset S\right\} .
$$

We call the set function $\psi_{s}^{-1}: \mathscr{P}(X) \rightarrow \mathscr{P}(X)$, the strong predecessor operator.

Similarly, we define the weak pre-image set of $S$ as,

$$
\psi_{w}^{-1}(S)=\{x \in X: \psi(x) \cap S \neq \emptyset\} .
$$

Also, we define the $k$-th weak pre-image set in similar fashion.

$$
\psi_{w}^{-k}(S)=\left\{x \in X: \psi^{k}(x) \cap S \neq \emptyset\right\} .
$$

Let us call the set function $\psi_{w}^{-1}: \mathscr{P}(X) \rightarrow \mathscr{P}(X)$, the weak predecessor operator. 
In the viewpoint of the DCDS, the strong and the weak pre-image sets of the MVIDS given by the multiple valued self-map $\psi \in \mathscr{M}\left(X^{X}\right)$ can be viewed as follows.

Remark 4.5. The strong pre-image set $\psi_{s}^{-1}(S)$ consists of the input states that must yield the outputs in $S$, while the weak pre-image set $\psi_{w}^{-1}(S)$ consists of the input states that can yield the outputs in $S$.

Remark 4.5 tells us that the strong pre-images can be used for predictive control problems, where one looks for input states that ensure the desired outputs; while the weak pre-images are suitable for investigative control problems, where one looks for all possible input states that could have caused the current state.

Another interesting distinction between the strong and the weak predecessor operators is that the latter operator $\psi_{w}^{-1}$ is a multiple valued self-map in $X$, while the latter operator $\psi_{s}^{-1}$ is not [10]. The fact that $\psi_{w}^{-1} \in \mathscr{M}\left(X^{X}\right)$ will turn out useful when characterizing the full-invariance of MVIDS (Theorem $5.4)$.

\subsection{Invariant sets of multiple valued iterative dynamical systems}

We are now ready to clarify the invariance concepts of MVID models.

Definition 4.6 (Invariant Sets of MVIDS). Let $X$ be a nonempty set and $\psi \subset \mathscr{M}\left(X^{X}\right)$.

A. We say $S \subset X$ is strongly quasi-invariant (positive-invariant) under $\psi$ if

$$
S \subset \psi_{s}^{-1}(S) .
$$

B. We say $S \subset X$ is weakly quasi-invariant (positive-invariant) under $\psi$ if

$$
S \subset \psi_{w}^{-1}(S) .
$$

C. We say $S \subset X$ is strongly invariant (full-invariant) under $\psi$ if

$$
S \subset \psi(S) \cap \psi_{s}^{-1}(S)
$$

D. We say $S \subset X$ is weakly invariant (full-invariant) under $\psi$ if

$$
S \subset \psi(S) \cap \psi_{w}^{-1}(S)
$$

When there is no danger of confusion, we will abuse the terms invariance and invariant sets to denote all of the above at the same time. When it becomes necessary, on the contrary, we will use the terms full-invariance to emphasize the distinction from the quasi-invariance.

The following proposition states that the inequalities (4.8) and (4.10) in Definition 4.6 can be expressed in more conservative forms as in SVIDS case (c.f. Definition 3.1). 
Proposition 4.7 (Alternative Definitions of Strong Invariance and Strong Quasi-invariance). Let $X$ be a nonempty set and $\psi \subset \mathscr{M}\left(X^{X}\right)$. Let $S \subset X$ and $S \neq \emptyset$. Then, $S$ is strongly quasi-invariant under $\psi$ if and only if $\psi(S) \subset S$, and it is strongly invariant under $\psi$ if and only if $\psi(S)=S$.

Proof. The equivalence relation on the strong quasi-invariance can be proved as follows.

$$
\begin{aligned}
S \subset \psi_{s}^{-1}(S) & \Longleftrightarrow \forall x \in S, \psi(x) \subset S \\
& \Longleftrightarrow \psi(S) \subset S .
\end{aligned}
$$

Now, combining the equivalence relation (4.12) and the set-inequality (4.10), we get the following result.

$$
\begin{aligned}
S \subset \psi(S) \cap \psi_{s}^{-1}(S) & \Longleftrightarrow S \cap \psi(S) \text { and } S \subset \psi_{s}^{-1}(S) \\
& \Longleftrightarrow S \cap \psi(S) \text { and } \psi(S) \subset S \\
& \Longleftrightarrow \psi(S)=S .
\end{aligned}
$$

Hence, the equivalence relation on the strong full-invariance follows.

Another interesting observation one can make is the following connection between the strong quasi-invariance and the weak predecessor.

Proposition 4.8 (The Insulation Condition [12]). Let $X$ be a nonempty set and $\psi \subset \mathscr{M}\left(X^{X}\right)$. Let $S \subset X$ and $S \neq \emptyset$. Then $X \backslash S$ is strongly quasiinvariant under $\psi$ if and only if $\psi_{w}^{-1}(S) \subset S$.

Proposition 4.8 will not be used to prove the main results of this paper, so we omit its proof for brevity. For more detail, see [12].

\subsection{Characterizing invariant sets}

The first main theorem of this paper is the characterization of various types of the invariant sets of MVIDS, in terms of the dynamical behavior of the system.

Theorem 4.9 (The Characterization Theorem for Invariance). Let $X$ be a nonempty set and $\psi \in \mathscr{M}\left(X^{X}\right)$. Let $S \subset X$.

A (Strong Quasi-invariance). The following conditions are equivalent.

$$
\begin{aligned}
& S \subset \psi_{s}^{-1}(S) . \\
& \psi(S) \subset S . \\
& x_{0} \in S \Rightarrow \forall k \in \mathbb{Z}^{+}, \psi^{k}\left(x_{0}\right) \subset S . \\
& S=\mathcal{F}_{s}^{\omega}(S) .
\end{aligned}
$$

B (Weak Quasi-invariance). The following conditions are equivalent.

$$
S \subset \psi_{w}^{-1}(S) \text {. }
$$




$$
\begin{aligned}
& x_{0} \in S \Rightarrow \forall k \in \mathbb{Z}^{+}, \psi^{k}\left(x_{0}\right) \cap S \neq \emptyset . \\
& S=\mathcal{F}_{w}^{\omega}(S) .
\end{aligned}
$$

C (Strong Full-invariance). The following conditions are equivalent.

$$
\begin{aligned}
& S \subset \psi(S) \cap \psi_{s}^{-1}(S) . \\
& \psi(S)=S . \\
& x_{0} \in S \Rightarrow \forall k \in \mathbb{Z}^{+}, \psi^{k}\left(x_{0}\right) \subset S \text { and } \psi_{w}^{-k}\left(x_{0}\right) \cap S \neq \emptyset . \\
& S=\mathcal{F}_{s}^{\omega}(S) \cap \mathcal{B}^{\omega}(S) .
\end{aligned}
$$

D (Weak Full-invariance). The following conditions are equivalent.

$$
\begin{aligned}
& S \subset \psi(S) \cap \psi_{w}^{-1}(S) . \\
& x_{0} \in S \Rightarrow \forall k \in \mathbb{Z}^{+}, \psi^{k}\left(x_{0}\right) \cap S \neq \emptyset \text { and } \psi_{w}^{-k}\left(x_{0}\right) \cap S \neq \emptyset . \\
& S=\mathcal{F}_{w}^{\omega}(S) \cap \mathcal{B}^{\omega}(S) .
\end{aligned}
$$

Proof of Theorem 4.9.A. The equivalence between (4.13) and (4.14) is established in Proposition 4.7. Assume (4.14) and let $x_{0} \in S$. Then, $\psi^{1}\left(x_{0}\right) \subset S$ from (4.14). Applying $\psi$ again, we get $\psi^{2}\left(x_{0}\right)=\psi\left(\psi\left(x_{0}\right)\right) \subset \psi(S) \subset S$. Repeating this process inductively, we get (4.15).

Now, assume (4.15). Because $\mathscr{O}^{+}\left(x_{0}: S\right) \subset \mathscr{O}^{+}\left(x_{0}: X\right)$ is always true, we need only to prove the other inclusion. Let $x_{0} \in S$ and $\left(x_{k}\right)_{k=0}^{\infty} \in \mathscr{O}^{+}\left(x_{0}: X\right)$. Then, $x_{1} \in \psi\left(x_{0}\right) \subset S$. Applying $\psi$ again, we get $x_{2} \in \psi\left(x_{1}\right) \subset \psi^{2}\left(x_{0}\right) \subset S$. Repeating this process inductively, we get $x_{k} \in \psi^{k}\left(x_{0}\right) \subset S$ for all $k \in \mathbb{Z}^{+}$. Hence, $\left(x_{k}\right)_{k=0}^{\infty} \in \mathscr{O}^{+}\left(x_{0}: S\right)$, and thus, (4.16) follows.

Finally, assume (4.16). Suppose that $S \not \subset \psi_{s}^{-1}(S)$. That is, for some $x_{0} \in S$, we can find $x_{1} \in \psi\left(x_{0}\right)$ but $x_{1} \notin S$. Choosing any $x_{2} \in \psi\left(x_{1}\right), x_{3} \in \psi\left(x_{2}\right)$, and so on, we get $\left(x_{k}\right)_{k=0}^{\infty} \subset \mathscr{O}^{+}\left(x_{0}: X\right) \backslash \mathscr{O}^{+}\left(x_{0}: S\right)$. This contradicts (4.16), and consequently (4.13) follows.

Proof of Theorem 4.9.B. Assume (4.17) and let $x_{0} \in S$. Then, $x_{0} \in S \subset$ $\psi_{w}^{-1}(S)$, so there exists a certain $x_{1} \in \psi\left(x_{0}\right) \cap S$. Do the same to $x_{1} \in S$ to get $x_{2} \in \psi\left(x_{1}\right) \cap S$. Repeating this process, we get an infinite forward orbit-chain $\left(x_{k}\right)_{k=0}^{\infty} \in \mathscr{O}^{+}\left(x_{0}: S\right)$, and thus (4.19) follows. Now, assume (4.19), that is, there exists a certain $\left(x_{k}\right)_{k=0}^{\infty} \in \mathscr{O}^{+}\left(x_{0}: S\right)$. Consequently, $x_{k} \in \psi^{k}\left(x_{0}\right)$ for each $k \in \mathbb{Z}^{+}$, and thus (4.18) follows. Finally, assume (4.18). Then for any $x_{0} \in S$, we must have $\psi\left(x_{0}\right) \cap S \neq \emptyset$, and thus $x_{0} \in \psi_{w}^{-1}(S)$. Since $x_{0}$ is arbitrary, (4.17) follows.

Proof of Theorem 4.9.C. Proposition 4.7 proves the equivalence between (4.20) and (4.21). Assume (4.20) and let $x_{0} \in S$. Then, we must have $\mathscr{O}^{+}\left(x_{0}: S\right)=$ $\mathscr{O}^{+}\left(x_{0}: X\right)$ for all $k \in \mathbb{Z}^{+}$because of Theorem 4.9.A. Furthermore, due to the fact that $S \subset \psi(S)$, there exists a certain $x_{-1} \in S$ such that $x_{0} \in \psi\left(x_{-1}\right)$. Applying the same argument, we cain find $x_{-2} \in S$ such that $x_{-1} \in \psi\left(x_{-2}\right)$. 
Repeating this process, we get an infinite backward orbit-chain $\left(x_{-k}\right)_{k=0}^{\infty}$ in $S$, and thus, $\mathscr{O}^{-}\left(x_{0}: S\right) \neq \emptyset$. Hence, (4.23) follows.

Next, we assume (4.23) and let $x_{0} \in S$. Then, $\mathscr{O}^{+}\left(x_{0}: S\right)=\mathscr{O}^{+}\left(x_{0}: X\right)$ implies $\psi^{k}\left(x_{0}\right) \subset S$ for all $k \in \mathbb{Z}^{+}$, as we saw in the proof of Theorem 4.9.A. The second part, $\mathscr{O}^{-}\left(x_{0}: S\right) \neq \emptyset$ implies that there exists an infinite backward orbitchain $\left(x_{-k}\right)_{k=0}^{\infty}$ in $S$. Hence, for all $k \in \mathbb{Z}^{+}$, we must have $x_{0} \in \psi^{k}\left(x_{-k}\right) \cap S$, and thus, $x_{-k} \in \psi_{w}^{-1}\left(x_{0}\right) \cap S \neq \emptyset$. Consequently, we get (4.22).

Finally, let use assume (4.22). Choose any $x_{0} \in S$. Then, $x_{0} \in \psi_{s}^{-1}(S)$, because $S \subset \psi_{s}^{-1}(S)$ (Theorem 4.9.A). Also, because of $\psi_{w}^{-1}\left(x_{0}\right) \cap S \neq \emptyset$, there must be a certain $x_{-1} \in S$ such that $x_{0} \in \psi\left(x_{-1}\right)$. Hence, $x_{0} \in \psi(S)$. Because $x_{0}$ was selected arbitrarily, we conclude that $S \subset \psi(S)$, and thus, (4.20) follows.

Proof of Theorem 4.9.D. Assume (4.24) and let $x_{0} \in S$. From Theorem 4.9.B, we get $\mathscr{O}^{+}\left(x_{0}: S\right) \neq \emptyset$. From the other condition, $S \subset \psi(S)$, we can find $x_{-1} \in S$ such that $x_{0} \in \psi\left(x_{-1}\right)$. From the same argument, we get $x_{-2} \in S$ such that $x_{-1} \in \psi\left(x_{-2}\right)$. Repeating this process, we get an infinite backward orbit-chain $\left(x_{-k}\right)_{k=0}^{\infty}$ in $S$. Hence, $\mathscr{O}^{-}\left(x_{0}: S\right) \neq \emptyset$, as well, and thus, (4.26) follows.

The proof that (4.26) implies (4.25) is similar to the corresponding step of the proof of Theorem 4.9.C.

For the final step, assume (4.25). We already saw that $S \subset \psi_{w}^{-1}(S)$ when proving Theorem 4.9.B. Choose any $x_{0} \in S$. Then, there must be a certain $x_{-1} \in \psi_{w}^{-1}(S) \cap S$, because the latter is nonempty. Thus, we get $x_{0} \in \psi\left(x_{-1}\right) \subset$ $\psi(S)$. Hence, (4.24) follows.

\section{Maximal invariant sets of multiple valued iterative dynamical systems}

\subsection{Maximal invariant sets}

We define the maximal invariant sets of MVIDS by generalizing Definition 3.3 as follows.

Definition 5.1 (Maximal Invariant Sets of MVIDS). Let $X$ be a nonempty set and $\psi \in \mathscr{M}\left(X^{X}\right)$. Let $Y \subset X$ and $Y \neq \emptyset$.

A. The strong locally maximal quasi-invariant subset $\mathcal{M}_{s}^{+}(Y)$ of $Y$ under $\psi$ is defined by the union of all strongly quasi-invariant subsets of $Y$. That is,

$$
\mathcal{M}_{s}^{+}(Y)=\bigcup\left\{S \subset Y: S \subset \psi_{s}^{-1}(S)\right\} .
$$

B. The weak locally maximal quasi-invariant subset $\mathcal{M}_{w}^{+}(Y)$ of $Y$ under $\psi$ is defined by the union of all weakly quasi-invariant subsets of $Y$. That is,

$$
\mathcal{M}_{w}^{+}(Y)=\bigcup\left\{S \subset Y: S \subset \psi_{w}^{-1}(S)\right\} .
$$


C. The strong locally maximal invariant subset $\mathcal{M}_{S}(Y)$ of $Y$ under $\psi$ is defined by the union of all strongly invariant subsets of $Y$. That is,

$$
\mathcal{M}_{s}(Y)=\bigcup\left\{S \subset Y: S \subset \psi(S) \cap \psi_{s}^{-1}(S)\right\} .
$$

D. The weak locally maximal invariant subset $\mathcal{M}_{w}(Y)$ of $Y$ under $\psi$ is defined by the union of all weakly invariant subsets of $Y$. That is,

$$
\mathcal{M}_{w}(Y)=\bigcup\left\{S \subset Y: S \subset \psi(S) \cap \psi_{w}^{-1}(S)\right\} .
$$

When $Y=X$, then we drop the term locally from Definition 5.1, or sometimes replace it with globally. Again, when there is no danger of confusion, we abuse the plural term maximal invariant sets to denote all of them at the same time.

The following lemma justifies our selection of the terms.

Lemma 5.2 (The Justification Lemma for Maximal Invariance). Let $X$ be a nonempty set and $\psi \in \mathscr{M}\left(X^{X}\right)$. Then given non-empty subset $Y$ of $X$, the strong/weak locally maximal quasi-invariant/full-invariant subsets of $Y$ are indeed maximal (in terms of the set inclusion) and strong/weak quasiinvariant/full-invariant.

Sketch of Proof. It is obvious that $\mathcal{M}_{s}^{+}(Y), \mathcal{M}_{w}^{+}(Y), \mathcal{M}_{s}(Y), \mathcal{M}_{w}(Y) \subset Y$ and that they are indeed maximal in terms of the set inclusion, because they were defined by the union of all strongly/weakly quasi-invariant/full-invariant subsets of $Y$. All there is left to prove, therefore, is the invariance.

$$
\begin{aligned}
x \in \mathcal{M}_{s}^{+}(Y) & \Rightarrow x \in S, \text { where } \exists S \subset Y, \psi(S) \subset S \\
& \Rightarrow \psi(x) \subset \psi(S) \subset S \subset \mathcal{M}_{s}^{+}(Y) . \\
x \in \mathcal{M}_{w}^{+}(Y) & \Rightarrow x \in S, \text { where } S \subset Y, S \subset \psi_{w}^{-1}(S) \\
& \Rightarrow \emptyset \neq \psi(x) \cap S \subset \psi(x) \cap \mathcal{M}_{w}^{+}(Y) . \\
x \in \mathcal{M}_{s}(Y) & \Rightarrow x \in S, \text { where } S \subset Y, S \subset \psi(S) \\
& \Rightarrow \exists y \in Y \text { such that } x \in \psi(y) \subset \psi\left(\mathcal{M}_{s}(Y)\right) . \\
x \in \mathcal{M}_{w}(Y) & \Rightarrow x \in S, \text { where } S \subset Y, S \subset \psi(S) \\
& \Rightarrow \exists y \in Y \text { such that } x \in \psi(y) \subset \psi\left(\mathcal{M}_{w}(Y)\right) .
\end{aligned}
$$

The statements (5.5) and (5.6) prove the strong and the weak quasi-invariance, respectively. Combining them with the latter statements (5.7) and (5.8), we get the full-invariance.

We conclude this subsection with the following obvious observation.

Proposition 5.3. Let $X$ and $Y$ be nonempty sets such that $Y \subset X$. Let $\psi \in \mathscr{M}\left(X^{X}\right)$. Then, $\mathcal{M}_{s}(Y)=\mathcal{M}_{s}\left(\mathcal{M}_{s}^{+}(Y)\right)$ and $\mathcal{M}_{w}(Y)=\mathcal{M}_{w}\left(\mathcal{M}_{w}^{+}(Y)\right)$. 
Sketch of Proof. The $\supset$ part is obvious. The $\subset$ part follows from the fact that each strong/weak full-invariant subset of $Y$ is strong/weak quasi-invariant as well, and thus belong to $\mathcal{M}_{s}^{+}(Y)$ or $\mathcal{M}_{w}^{+}(Y)$ as well.

\subsection{Characterizing maximal invariant sets}

The second main result of this paper is the characterization theorem of the maximal invariant sets. As in Theorem 4.9, we characterize them in terms of the dynamics of the system.

Theorem 5.4 (The Characterization Theorem for Maximal Invariance). Let $X$ be a nonempty set and $\psi \in \mathscr{M}\left(X^{X}\right)$. Then, given non-empty subset $Y$ of $X$, we get the following results.

$$
\begin{aligned}
& \mathcal{M}_{s}^{+}(Y)=\mathcal{F}_{s}^{\omega}(Y) . \\
& \mathcal{M}_{w}^{+}(Y)=\mathcal{F}_{w}^{\omega}(Y) . \\
& \mathcal{M}_{s}(Y)=\mathcal{B}^{\omega}\left(\mathcal{F}_{s}^{\omega}(Y)\right) . \\
& \mathcal{M}_{w}(Y)=\mathcal{B}^{\omega}\left(\mathcal{F}_{w}^{\omega}(Y)\right)=\mathcal{B}^{\omega}(Y) \cap \mathcal{F}_{w}^{\omega}(Y) .
\end{aligned}
$$

Proof of Theorem 5.4, Part 1: Equality (5.9). First, assume $x_{0} \in \mathcal{M}_{s}^{+}(Y)$, that is, there is a certain strongly quasi-invariant subset $S$ of $Y$ such that $x_{0} \in$ $S$. Then, from Theorem 4.9.A and Proposition 4.2, we conclude that $x_{0} \in$ $\mathcal{F}_{s}^{\omega}(S) \subset \mathcal{F}_{s}^{\omega}(Y)$. Hence, we get the first inclusion, $\mathcal{M}_{s}^{+}(Y) \subset F_{s}^{\omega}(Y)$.

Conversely, assume $x_{0} \in \mathcal{F}_{s}^{\omega}(Y)$, that is, every infinite forward orbit-chain of $x_{0}$ in $X$ must stay in $Y$. Choose any $x_{1} \in \psi\left(x_{0}\right) \subset \psi\left(\mathcal{F}_{s}^{\omega}(Y)\right)$. Then, every infinite forward-orbit-chain $\left(x_{k}\right)_{k=1}^{\infty}$ of $x_{1}$ in $X$ gives rise to the infinite forward orbit-chain $\left(x_{k}\right)_{k=0}^{\infty}$, which must stay in $Y$. Therefore, $\mathscr{O}^{+}\left(x_{1}: X\right)=$ $\mathscr{O}^{+}\left(x_{1}: Y\right)$, and thus, $x_{1} \in \mathcal{F}_{s}^{\omega}(Y)$. Because the selection of $x_{0} \in \mathcal{F}_{s}^{\omega}(Y)$ and $x_{1} \in \psi\left(x_{0}\right)$ was arbitrary, we conclude that $\psi\left(\mathcal{F}_{s}^{\omega}(Y)\right) \subset \mathcal{F}_{s}^{\omega}(Y)$, that is, $\mathcal{F}_{s}^{\omega}(Y)$ is a strongly quasi-invariant subset of $Y$, and thus is must be contained in the locally maximal quasi-invariant subset $\mathcal{M}_{s}^{+}(Y)$. Hence, we get the other direction, $\mathcal{F}_{s}^{\omega}(Y) \subset \mathcal{M}_{s}^{+}(Y)$, as well.

Proof of Theorem 5.4, Part 2: Equality (5.10). It is easy to see that the inclusion $\mathcal{M}_{w}^{+}(Y) \subset \mathcal{F}_{w}^{\omega}(Y)$ follows from Theorem 4.9.B, just as $\mathcal{M}_{s}^{+}(Y) \subset \mathcal{F}_{w}^{\omega}(Y)$ followed from Theorem 4.9.A. Let us consider the other direction, now.

Let $x_{0} \in F_{w}^{\omega}(Y)$, that is, there exists a certain infinite forward orbitchain $\left(x_{k}\right)_{k=0}^{\infty}$ in $Y$. Then, we can easily see that $x_{1} \in \psi\left(x_{0}\right) \cap F_{w}^{\omega}(Y)$, because $\left(x_{k}\right)_{k=1}^{\infty} \in \mathscr{O}^{+}\left(x_{1}: Y\right)$. Therefore, $\psi\left(x_{0}\right) \cap F_{w}^{\omega}(Y) \neq \emptyset$, and thus $x_{0} \in \psi_{w}^{-1}\left(F_{w}^{\omega}(Y)\right)$. Because the selection of $x_{0}$ was arbitrary, we conclude that $F_{w}^{\omega}(Y) \subset \psi_{w}^{-1}\left(F_{w}^{\omega}(Y)\right)$, and thus, $F_{w}^{\omega}(Y) \subset \mathcal{M}_{w}^{+}(Y)$.

Proof of Theorem 5.4, Part 3: Equality (5.11). From (5.9) and Proposition 5.3, we get

$$
\mathcal{M}_{s}(Y)=\mathcal{M}_{s}\left(\mathcal{M}_{s}^{+}(Y)\right)=\mathcal{M}_{s}\left(\mathcal{F}_{s}^{\omega}(Y)\right)
$$


We need only to prove, therefore,

$$
\mathcal{M}_{s}(Z)=\mathcal{B}^{\omega}(Z)
$$

when $Z$ is strongly quasi-invariant.

The inclusion $\mathcal{M}_{s}(Z) \subset \mathcal{B}^{\omega}(Z)$ follows from Theorem 4.9.C, through the argument similar to the corresponding parts of the proof of (5.9) and (5.10). We now show the other inclusion by proving that $\mathcal{B}^{\omega}(Z)$ is strongly invariant, and thus, included in the strong maximal invariant set.

Let $x_{0} \in \mathcal{B}^{\omega}(Z)$, where $\psi(Z) \subset Z$. That is, $\left(x_{-k}\right)_{k=0}^{\infty} \in \mathscr{O}^{-}\left(x_{0}: Z\right)$. Choose any $x_{1} \in \psi\left(x_{0}\right)$. Then, $\left(x_{-k}\right)_{k=-1}^{\infty} \in \mathscr{O}^{-}\left(x_{1}: Z\right)$, because $Z$ is strongly quasiinvariant. Hence, $x_{1} \in \mathcal{B}^{\omega}(Z)$. Because this holds for all $x_{1} \in \psi\left(x_{0}\right)$ for any $x_{0} \in \mathcal{B}^{\omega}(Z)$, we must have

$$
\mathcal{B}^{\omega}(Z) \subset \psi_{s}^{-1}\left(\mathcal{B}^{\omega}(Z)\right)
$$

Furthermore, starting the infinite backward orbit-chain from $x_{-1}$, we get

$$
\left(x_{-k}\right)_{k=1}^{\infty} \in \mathscr{O}^{-}\left(x_{-1}: Z\right) \text {. }
$$

Therefore, $x_{0} \in \psi\left(x_{-1}\right) \subset \psi\left(\mathcal{B}^{\omega}(Z)\right)$. Since $x_{0}$ is arbitrary, we get

$$
\mathcal{B}^{\omega}(Z) \subset \psi\left(\mathcal{B}^{\omega}(Z)\right) \text {. }
$$

Combining (5.15) and (5.16), we get the strong quasi-invariance of $\mathcal{B}^{\omega}(Z)$. Consequently, we get the other inclusion, $\mathcal{M}_{s}(Z) \supset \mathcal{B}^{\omega}(Z)$, too. This complete the proof of (5.14). The desired result, (5.11), follows from (5.9), (5.13), (5.14), and the fact that $\mathcal{F}_{s}^{\omega}(Y)$ is strongly quasi-invariant (Lemma 5.2).

Proof of Theorem 5.4, Part 4: Equality (5.12). It is easy to see that

$$
\begin{aligned}
\mathcal{M}_{w}(Y) & =\mathcal{M}_{w}\left(\mathcal{M}_{w}^{+}(Y)\right) & & \because \text { Proposition } 5.3, \\
& =\mathcal{M}_{w}\left(\mathcal{F}_{w}^{\omega}(Y)\right) & & \because \text { Equality }(5.10), \\
& \subset \mathcal{B}^{\omega}\left(\mathcal{F}_{w}^{\omega}(Y)\right) & & \because \text { Theorem 4.9.D, } \\
& \subset \mathcal{B}^{\omega}(Y) \cap \mathcal{F}_{w}^{\omega}(Y) & & \because \text { Proposition } 4.2 \& \text { Lemma 4.3. }
\end{aligned}
$$

We will show the other inclusion by proving $\mathcal{B}^{\omega}(Y) \cap \mathcal{F}_{w}^{\omega}(Y)$ is weakly invariant.

Suppose $x_{0} \in \mathcal{B}^{\omega}(Y) \cap \mathcal{F}_{w}^{\omega}(Y)$. In particular, $x_{0} \in F_{w}^{\omega}(Y)$. Now, $\mathcal{F}_{w}^{\omega}(Y)$ is weakly quasi-invariant because of (5.10) and Lemma 5.2. Therefore, there exists a certain $x_{1} \in Y$ such that

$$
x_{1} \in \psi\left(x_{0}\right) \cap \mathcal{F}_{w}^{\omega}(Y) .
$$

Now, because $x_{0} \in \mathcal{B}^{\omega}(Y)$, there is a certain infinite backward orbit-chain $\left(x_{-k}\right)_{k=0}^{\infty}$ in $Y$. Starting from $x_{1}$, therefore, we get $\left(x_{-k}\right)_{k=-1}^{\infty} \in \mathscr{O}^{-}\left(x_{1}: Y\right)$. That is,

$$
x_{1} \in \psi\left(x_{0}\right) \cap \mathcal{B}^{\omega}(Y) .
$$

Combining (5.17) and (5.18), we get $\psi\left(x_{0}\right) \cap\left(\mathcal{B}^{\omega}(Y) \cap \mathcal{F}_{w}^{\omega}(Y)\right) \neq \emptyset$. Or,

$$
x_{0} \in \psi_{w}^{-1}\left(\mathcal{B}^{\omega}(Y) \cap \mathcal{F}_{w}^{\omega}(Y)\right) .
$$


On the other hand, because $x_{0} \in \mathcal{B}^{\omega}(Y)$, we can find a certain $x_{-1} \in Y$ such that $x_{0} \in \psi\left(x_{-1}\right)$. Starting the forward and the backward iterations of $\psi$ from $x_{-1}$, we get the infinite forward and the backward orbit-chains, $\left(x_{k}\right)_{k=-1}^{\infty} \in \mathscr{O}^{+}\left(x_{-1}: Y\right)$ and $\left(x_{-k}\right)_{k=1}^{\infty} \in \mathscr{O}^{-}\left(x_{-1}: Y\right)$. Therefore,

$$
x_{0} \in \psi\left(x_{-1}\right) \subset \psi\left(\mathcal{B}^{\omega}(Y) \cap \mathcal{F}_{s}^{\omega}(Y)\right) .
$$

Because $x_{0}$ was selected arbitrarily, the combination of (5.19) and (5.20) yields,

$$
\mathcal{B}^{\omega}(Y) \cap \mathcal{F}_{w}^{\omega}(Y) \subset \psi_{w}^{-1}\left(B^{\omega}(Y) \cap \mathcal{F}_{w}^{\omega}(Y)\right) \cap\left(B^{\omega}(Y) \cap \mathcal{F}_{w}^{\omega}(Y)\right) .
$$

That is, $\mathcal{B}^{\omega}(Y) \cap \mathcal{F}_{w}^{\omega}(Y)$ is weakly invariant. Hence, the desired inclusion $B^{\omega}(Y) \cap \mathcal{F}_{w}^{\omega}(Y) \subset \mathcal{M}_{w}(Y)$ follows.

\subsection{Controllability problems of locally maximal invariant sets}

The third main result of this paper is to set up the $\infty$-step controllability problems of the maximal invariant sets and prove that their well-posedness. Our definitions of the maximal invariant sets in Definition 5.1 ensures the existence, as well as the maximality and the invariance, in contrast to some other methods $[4,7,8,19]$. However, Definition 5.1 does not ease the difficulty in identifying or approximating the maximal invariant sets at all. Theorem 5.4 helps, but following the orbit-chains of large number of (possibly infinitely many or even uncountably many) individual points is still too much to handle in any realistic applications. Theorem 5.7 resolves this difficulty, by providing iterative methods that yield the desired maximal invariant sets. We need, however, the following definition and lemma before expressing and proving Theorem 5.7.

Definition 5.5 (Controllable/Reachable Sets). Let $X$ be a nonempty set and $\psi \in \mathscr{M}\left(X^{X}\right)$. Let $Z \subset Y \subset X$. We define the $n$-step strongly controllable set $Y_{s}^{-n}$, the $n$-step weakly controllable set $Y_{w}^{-n}$, and the $n$-step reachable set $Y^{n}$, of $Y$ as follows.

$$
\begin{array}{ll}
Y_{s}^{0}=Y, \quad Y_{s}^{-n}=Y \cap \psi_{s}^{-1}\left(Y_{s}^{n-1}\right), \\
Y_{w}^{0}=Y, \quad Y_{w}^{-n}=Y \cap \psi_{w}^{-1}\left(Y_{s}^{n-1}\right), \\
Y^{0}=Y, \quad Y^{n}=Y \cap \psi\left(Y^{n-1}\right),
\end{array}
$$

where $n \in \mathbb{Z}^{+}$. Furthermore, let us denote

$$
Y_{s}^{-\infty}=\bigcap_{n=0}^{\infty} Y_{s}^{-n}, \quad Y_{w}^{-\infty}=\bigcap_{n=0}^{\infty} Y_{w}^{-n}, \quad Y^{\infty}=\bigcap_{n=0}^{\infty} Y^{n}
$$

Lemma 5.6 (The Justification Lemma for Controllability/Reachability). Let $X$ be a nonempty set and $\psi \in \mathscr{M}\left(X^{X}\right)$. Then, given $Y \subset X$, the following results hold.

$$
\begin{array}{ll}
Y_{s}^{-n}=\mathcal{F}_{s}^{n}(Y), & n \in\{0,1,2, \ldots\} . \\
Y_{w}^{-n}=\mathcal{F}_{w}^{n}(Y), \quad n \in\{0,1,2, \ldots\} .
\end{array}
$$




$$
Y_{w}^{n}=\mathcal{B}^{n}(Y), \quad n \in\{0,1,2, \ldots\} .
$$

Proof. We proceed with the induction. There is nothing to prove when $n=0$. Suppose that (5.25), (5.26) and (5.27) are true for $n=k-1$.

Now, note that $x_{0} \in Y_{s}^{-k}=Y \cap \psi_{s}^{-1}\left(Y^{-(k-1)}\right)=Y \cap \psi_{s}^{-1}\left(F_{s}^{k-1}(Y)\right)$ if and only if $x_{0} \in Y$ and $\psi\left(x_{0}\right) \subset F_{s}^{k-1}(Y)$. Now, $\psi\left(x_{0}\right) \subset F_{s}^{k-1}(Y)$ means that every forward iterates of every $x_{1} \in \psi\left(x_{0}\right)$ stays in $Y$ up to $k-1$ iterations. Starting the iteration from $x_{0} \in Y$, therefore, we get all the forward iterates $x_{0} \in Y$ up to $k$ iterations. Hence, we conclude that $x_{0} \in \mathcal{F}_{s}^{k}(Y)$ is equivalent to $x_{0} \in Y$ and $\psi\left(x_{0}\right) \subset F_{s}^{k-1}(Y)$, and consequently to $x_{0} \in Y_{s}^{-k}$. Since the selection of $x_{0}$ was arbitrary, we get $Y_{s}^{-k}=\mathcal{F}_{s}^{k}(Y)$. This proves (5.25).

Likewise, let us note that $x_{0} \in Y_{w}^{-k}=Y \cap \psi_{w}^{-1}\left(Y^{-(k-1)}\right)=Y \cap \psi_{w}^{-1}\left(F_{w}^{k-1}(Y)\right)$ if and only if $x_{0} \in Y$ and $\psi\left(x_{0}\right) \cap F_{s}^{k-1}(Y) \neq \emptyset$. That is, $x_{0} \in Y$ and there is a certain $\left(x_{1}, \ldots, x_{k}\right) \in \mathscr{O}^{k-1}\left(x_{1}: Y\right)$. This is the same as $\left(x_{0}, x_{1}, \ldots, x_{k}\right) \in$ $\mathscr{O}^{n}\left(x_{1}: Y\right)$. Hence, we conclude that $x_{0} \in \mathcal{F}_{w}^{k}(Y)$ is equivalent to $x_{0} \in Y$ and $\psi\left(x_{0}\right) \cap F_{s}^{k-1}(Y) \neq \emptyset$, and consequently to $x_{0} \in Y_{w}^{-k}$. Thus, $Y_{w}^{-k}=\mathcal{F}_{w}^{k}(Y)$ follows. This proves (5.26).

Finally, note that $x_{0} \in Y_{w}^{k}=Y \cap \psi\left(Y^{k-1}\right)=Y \cap \psi\left(\mathcal{B}^{k-1}(Y)\right)$ if and only if $x_{0} \in Y$ and $x_{0} \in \psi\left(x_{-1}\right)$ where $x_{-1} \in \mathcal{B}^{k-1}(Y)$. This is the same as the existence of a backward orbit-chain $\left(x_{0}, x_{-1}, \ldots x_{-k}\right) \in \mathscr{O}^{-k}\left(x_{0}: Y\right)$, by starting the backward iteration from $x_{0} \in Y$. Hence, we get $x_{0} \in \mathcal{B}^{k}(Y)$ if and only if $x_{0} \in Y$ and $x_{0} \in \psi\left(x_{-1}\right) \subset \psi\left(\mathcal{B}^{k-1}(Y)\right)$, and consequently to $x_{0} \in Y_{w}^{k}$. Thus, we get $Y_{w}^{k}=\mathcal{B}^{k}(Y)$. This proves (5.27).

We are now ready to state and prove the third main theorem of this paper.

Theorem 5.7 (The Controllability Theorem). Let $X$ be a nonempty set and $\psi \in \mathscr{M}\left(X^{X}\right)$. Let $Y \subset X$.

A. For any $Y \subset X$,

$$
Y_{s}^{0} \supset Y_{s}^{-1} \supset Y_{s}^{-2} \supset \cdots \supset Y_{s}^{-\infty}=\mathcal{M}_{s}^{+}(Y) .
$$

B. Suppose that $\psi$ is finitely-many-valued in $Y$, that is, $\psi(y)$ is a finite set for all $y \in Y$. Then,

$$
Y_{w}^{0} \supset Y_{w}^{-1} \supset Y_{w}^{-2} \supset \cdots \supset Y_{w}^{-\infty}=\mathcal{M}_{w}^{+}(Y) .
$$

C. Suppose that $\psi_{w}^{-1}$ is finitely-many-valued in $Y$. Then,

$$
\left(Y_{s}^{0}\right)^{0} \supset\left(Y_{s}^{-1}\right)^{1} \supset\left(Y_{s}^{-2}\right)^{2} \supset \cdots \supset\left(Y_{s}^{-\infty}\right)^{\infty}=\mathcal{M}_{s}(Y) .
$$

D. Suppose that $\psi$ and $\psi_{w}^{-1}$ are both finitely-many-valued in $Y$. Then,

$$
Y \supset\left(Y^{1} \cap Y_{w}^{-1}\right) \supset\left(Y^{2} \cap Y_{w}^{-2}\right) \supset \cdots \supset\left(Y^{\infty} \cap Y_{w}^{-\infty}\right)=\mathcal{M}_{w}(Y) .
$$

Note that the equality (5.23) of Definition 5.5 was used in the part $\mathrm{C}$ of Theorem 5.7. That is, by putting $Y_{s}^{-i}$ in place of $Y$ in Equality (5.23), we get $\left(Y_{s}^{-i}\right)^{0}=Y_{s}^{-i}$ and $\left(Y_{s}^{-i}\right)^{j}=Y_{s}^{-i} \cap \psi\left(Y_{s}^{j-1}\right)$. Equality (5.30) includes only the 
spacial cases where $j=i$ for each $i$. All $i$ 's and $j$ 's must be considered in the proof, however.

Proof of Theorem 5.7, the first part. Lemma 5.6 and Theorem 5.4 tell us that the descending chains (5.28), (5.29) and (5.31) are the same as (4.1), (4.2) and (4.3) of Lemma 4.3, respectively, except for the last equalities of (5.29) and (5.31). This observation proves Theorem 5.7.A, and leaves only the following two equalities from completing the proof of Theorem 5.7.B and Theorem 5.7.D. They are,

$$
\mathcal{F}_{w}^{\infty}(Y)=\mathcal{F}_{w}^{\omega}(Y)
$$

and

$$
\mathcal{B}^{\infty}(Y)=\mathcal{B}^{\omega}(Y) .
$$

Note that Lemma 4.3 tells us that the equality (5.32) is equivalent to the last equality of (5.29), and the combination of the equalities (5.32) and (5.33) yields

$$
\mathcal{B}^{\infty}(Y) \cap \mathcal{F}_{w}^{\infty}(Y)=\mathcal{B}^{\omega}(Y) \cap \mathcal{F}_{w}^{\omega}(Y),
$$

which is equivalent to the last equality of (5.31).

Suppose that $\psi$ is finitely-many-valued in $Y$. Let $x_{0} \in \mathcal{F}_{s}^{\infty}(Y)$, that is, $\mathscr{O}^{n}\left(x_{0}: Y\right) \neq \emptyset$ for all $n \in \mathbb{Z}^{+}$. Choose one forward orbit-chain of $x_{0}$ of length $n$ in $Y$ for each $n \in \mathbb{Z}^{+}$. Among these infinitely many forward orbitchains of $x_{0}$ in $Y$, infinitely many of them must share the same first component $x_{1} \in \psi\left(x_{0}\right) \cap Y$, because $\psi$ is finitely-many-valued. These result in infinitely many forward orbit-chains of $x_{1}$ in $Y$. We can choose $x_{2} \in \psi\left(x_{1}\right) \cap Y$ through the same process, because $\psi\left(x_{1}\right)$ is a finite set. Because $\psi$ is finitely-manyvalued, we can repeat the same argument to get an infinite forward orbit-chain $\left(x_{k}\right)_{k=0}^{\infty} \in \mathscr{O}^{+}\left(x_{0}: Y\right)$. Therefore, $x_{0} \in \mathcal{F}_{w}^{\omega}(Y)$, and thus, $\mathcal{F}_{w}^{\infty}(Y) \subset \mathcal{F}_{w}^{\omega}(Y)$. Because the other direction is always true (Lemma 4.3), the equality (5.32) follows. This completes the proof of Theorem 5.7.B.

Now, suppose that both $\psi$ and $\psi_{w}^{-1}$ is finitely-many-valued. We already showed (5.32) when $\psi$ is finitely-many-valued. The proof of $(5.33)$ when $\psi_{w}^{-1}$ is finitely-many-valued is analogous to the former, where the only difference being the use of the backward orbit-chains instead of the forward orbit-chains. Consequently, we get the equality (5.34), and this completes the proof of Theorem 5.7.D.

The use of so called the infinite pigeon hole principle in the first part of the proof of Theorem 5.7 was inspired by [19].

Proof of Theorem 5.7, the second part. Suppose that $\psi$ is finitely-many-valued in $Y$. From the equalities (5.9) and (5.11) of Theorem 5.4 and the equality (5.33), we get

$$
\mathcal{M}_{s}(Y)=\mathcal{B}^{\omega}\left(\mathcal{M}_{s}^{+}(Y)\right)=\mathcal{B}^{\infty}\left(\mathcal{M}_{s}^{+}(Y)\right)
$$


Note that we replaced $Y$ by $\mathcal{M}_{s}^{+}(Y)$ in (5.33) to get the second equality of (5.35). We are allowed to do this, because $\mathcal{M}_{s}^{+}(Y) \subset Y$, and therefore, $\psi$ is finitely-many-valued in $\mathcal{M}_{s}^{+}(Y)$, too.

Applying Lemma 5.6 and (5.35) to Lemma 4.3, we get a descending chain,

$$
\mathcal{M}_{s}^{+}(Y)^{0} \supset \mathcal{M}_{s}^{+}(Y)^{1} \supset \mathcal{M}_{s}^{+}(Y)^{2} \supset \cdots \supset \mathcal{M}_{s}^{+}(Y)^{\infty}=\mathcal{M}_{s}(Y)
$$

Applying (5.28) to (5.36), we get the following double chain, where each arrow $(\rightarrow)$ stands for the set inclusion $(\supset)$ :

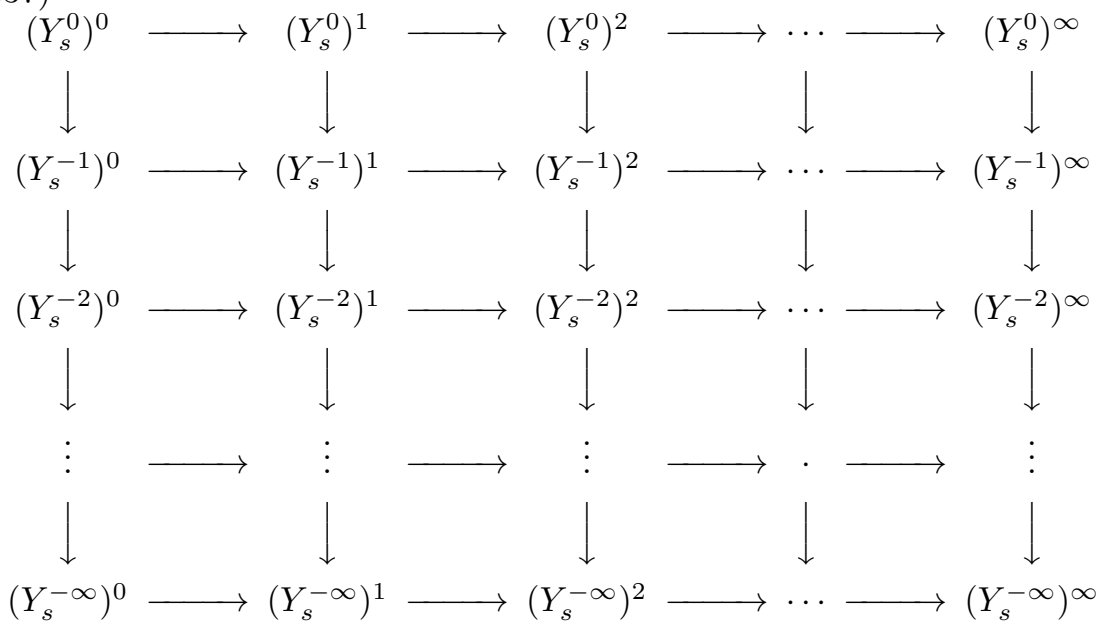

Taking the diagonal chain of the commutative diagram (5.30), we get the descending chain,

$$
\left(Y_{s}^{0}\right)^{0} \supset\left(Y_{s}^{-1}\right)^{1} \supset\left(Y_{s}^{-2}\right)^{2} \supset \cdots \supset\left(Y_{s}^{-\infty}\right)^{\infty}
$$

whose last entry satisfies $\left(Y_{s}^{-\infty}\right)^{\infty}=\mathcal{M}_{s}(Y)$, because of (5.28) and (5.36). This completes the proof of Theorem 5.7.C.

Theorem 5.7 tells us that the infinite-step controllability problem and the finite-approximate control problem are always well-posed for locally maximal strongly quasi-invariant sets, and gives us some sufficient conditions for those problems to be well-posed for the other maximal invariant sets. Also, if we disregard the approximation part by the descending chains of the finite-step controllable/reachable sets, we can re-express Theorem 5.7 in more concise (albeit less useful) form as follows.

Corollary 5.8. Let $X$ be a nonempty set and $\psi \in \mathscr{M}\left(X^{X}\right)$. Then, given $Y \subset X$, the following results hold.

$$
\mathcal{M}_{s}^{+}(Y)=\bigcap_{n=0}^{\infty} \psi_{s}^{-n}(Y) \text {. }
$$




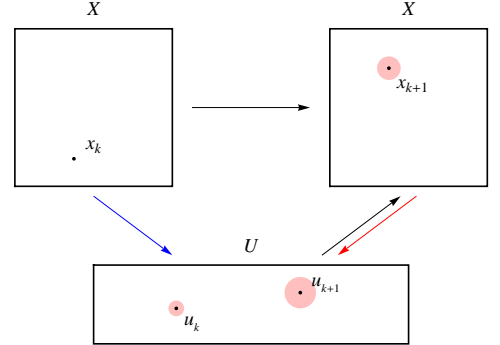

FiguRE 6.1

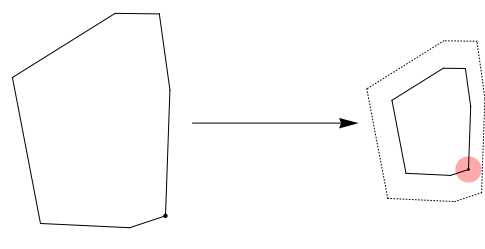

FiguRE 6.2

$$
\begin{aligned}
\mathcal{M}_{w}^{+}(Y) & =\bigcap_{n=0}^{\infty} \psi_{w}^{-n}(Y) . \\
\mathcal{M}_{s}(Y) & =\bigcap_{n=0}^{\infty} \psi^{n}\left(\bigcap_{k=0}^{n} \psi_{w}^{-k}(Y)\right) . \\
\mathcal{M}_{w}(Y) & =\bigcap_{n=0}^{\infty}\left(\psi^{n}(Y) \cap \psi_{w}^{-n}(Y)\right) .
\end{aligned}
$$

Proof. The proof of Corollary 5.8 follows from Theorem 5.7. It is elementary but tedious, so we leave it to the interested readers.

\section{Appendix: Examples}

In this section, we discuss some simple MVIDS-specific examples of the infinite-step controllability and finite-step approximate control problems of the maximal invariant sets. Let $X \subset \mathbb{R}^{n}$ be a phase space. Suppose that a map $f: X \rightarrow X$ is a linear contraction, so that the iteration of $f$ on $X$ would yield a single point. Let us consider two types of disturbances. Let the first disturbance be the random disturbance around the image points that blurs the outcomes (Figure 6.1). In terms of the set-dynamics, however, this disturbance can be regarded as nothing but a damping of the contraction (Figure 6.2). Now, let the second disturbance be the competition between more than one attractors.

Figure 6.3 and Figure 6.4 visualize the effects of the competing attractors and the maximal full-invariant set of this dynamics, respectively. It was possible to avoid the complication caused by the first disturbance, as illustrated in Figure 6.2. However, the second disturbance made the DCDS very difficult to investigate in conventional means. Figure 6.4 illustrates one such example that can be studied by MVID Modeling. In this simple example, by the way, the strong and weak maximal full-invariant sets are the same, so there is no need to consider both (5.30) and (5.31). Only the latter inequality was used to generate Figure 6.4. 


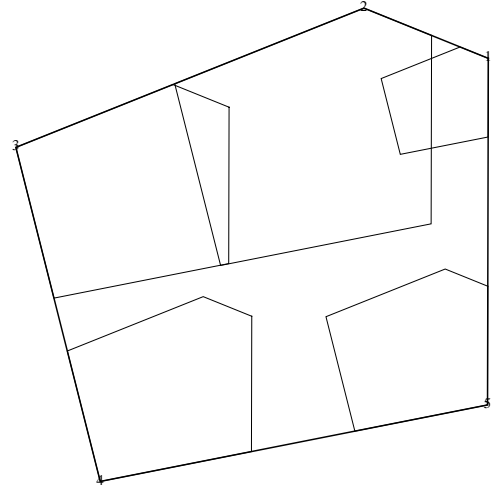

FigURE 6.3

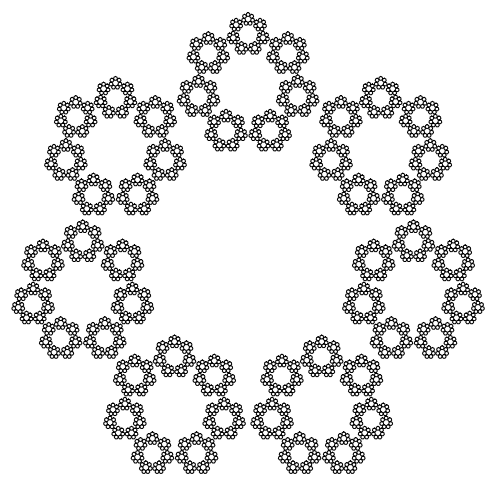

FiguRe 6.5

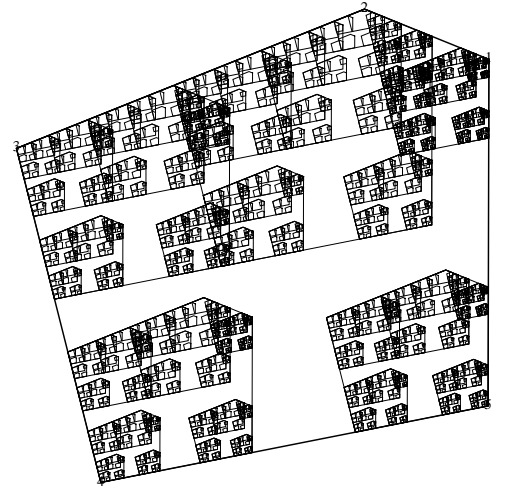

FigURE 6.4

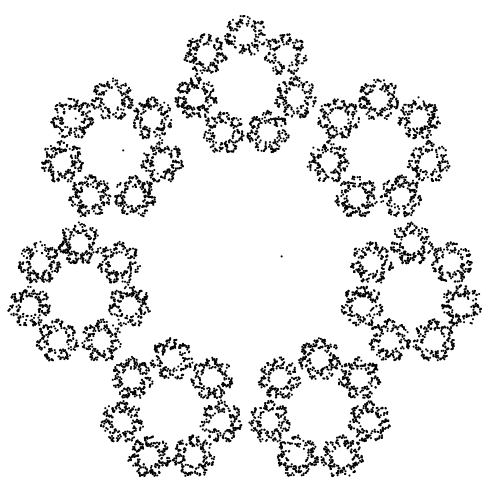

FiguRE 6.6

Figure 6.5 illustrates the dynamics and the maximal full invariant sets of a similar DCDS. In this example, the author placed 7 competing attractors symmetrically, and let the second disturbance be the random competing attractions.

In reality, of course, one cannot expect such a symmetry. The reason that the author considered an example with such stringent symmetry condition is in part aesthetic beauty, but more importantly, easy recognition. Compare Figure 6.5 and Figure 6.6. One can easily recognize that they both depict the same set, the maximal full-invariant set to be precise. Figure 6.5 was generated by the author's crude Mathematica program, and it took about 2.41001 seconds of CPU time of his personal computer, including Mathematica's initialization time. Figure 6.6, on the other hand, was generated by a forward orbit of the original DCDS. Even though the first disturbance was disregarded in order to reduce the run-time, the same computer took about 35.7192 seconds of CPU 
time to complete Figure 6.6. See [11] and [13] for more detail behind Figure 6.4 and Figure 6.5, respectively.

At least in some cases, the MVID modeling and the resulting set-dynamics appear to be useful tools, not only in pure mathematics but also in computational point of view as well. In general, however, the development of the computational algorithm for MVID models of DCDS is still in its primitive stage. At this moment, I do not know whether the successful improvement of the finite-step approximate control problem for the elementary example that we just discussed in this section can be duplicated or not for more realistic problems. Nonetheless, I believe this is a worthy topic that deserves our attention for the future research.

\section{References}

[1] R. Agarwal, M. Bohner, D. O'Regan, and A. Peterson, Dynamic equations on time scales: a survey, J. Comput. Appl. Math. 141 (2002), no. 1-2, 1-26.

[2] D. K. Arrowsmith and C. M. Place, An Introduction to Dynamical Systems, Cambridge University Press, 1990.

[3] Z. Arstein and S. Rakovic, Feedback invariance under uncertainty via set-iterates, Automatica 44 (2008), 520-525.

[4] P. Ashwin, X. C. Fu, T. Nishikawa, and K. Zyczkowski, Invariant sets for discontinuous parabolic area-preserving torus maps, Nonlinearity 13 (2000), no. 3, 819-835.

[5] F. Blanchini, Set invariance in control, Automatica J. IFAC 35 (1999), no. 11, 17471767.

[6] R. Findeisen, L. Imsland, F. Allgöwer, and B. A. Foss, State and output feedback nonlinear model predictive control: An overview, Euro. J. Control 9 (2003), no. 9, 179-195.

[7] X. C. Fu, F. Y. Chen, and X. H. Zhao, Dynamical properties of 2-torus parabolic maps, Nonlinear Dynam. 50 (2007), no. 3, 539-549.

[8] X. C. Fu and J. Duan, On global attractors for a class of nonhyperbolic piecewise affine maps, Phys. D 237 (2008), no. 24, 3369-3376.

[9] B. Kahng, Maximal invariant sets of multiple valued iterative dynamics in disturbed control systems, Int. J. Circuits, Systems and Signal Processing 2 (2008), 113-120.

[10] _ Positive invariance of multiple valued iterative dynamical systems in disturbed control models, in Proc. Med. Control Conf. Thessaloniki, Greece, 2009, pp. 663-668.

[11] _ The approximate control problems of the maximal invariant sets of non-linear discrete-time disturbed control dynamical systems: an algorithmic approach, in Proc. Int. Conf. on Control and Auto. and Sys. Gyeonggi-do, Korea, 2010, pp. 1513-1518.

[12] _ A survey of maximal invariance in multiple valued iterative dynamics models for nonlinear control and automation, preprint, 2012.

[13] B. Kahng and J. Davis, Maximal dimensions of uniform sierpinski fractals, Fractals 18 (2010), no. 4, 451-460.

[14] B. Kahng and M. Mendes, The controllability problems of the maximal invariant sets of discrete time dynamical systems, preprint, 2008.

[15] E. Kerrigan, J. Lygeros, and J. M. Maciejowski, A geometric approach to reachability computations for constrained discrete-time systems, in IFAC World Congress, Barcelona, Spain, 2002.

[16] E. Kerrigan and J. M. Maciejowski, Invariant sets for constrained nonlinear discretetime systems with application to feasibility in model predictive control, in Proc. 39th IEEE Conf. on Decision and Control, Sydney, Australia, 2000. 
[17] D. Q. Mayne, J. B. Rawlings, C. V. Rao, and P. O. M. Scokaert, Constrained model predictive control: stability and optimality, Automatica J. IFAC 36 (2000), no. 6, 789814.

[18] D. Q. Mayne, M. Seron, and S. V. Rakovic, Robust model predictive control of constrained linear systems with bounded disturbances, Automatica J. IFAC 41 (2005), no. $2,219-224$.

[19] M. Mendes, Dynamics of Piecewise Isometric Systems with Particular Emphasis to the Goetz Map, Ph. D. Thesis, University of Surrey, 2001.

[20] C. J. Ong and E. G. Gilbert, Constrained linear systems with disturbances: enlargement of their maximal invariant sets by nonlinear feedback, in Proc. Amer. Control Conf. Minneapolis, MN, 2006, pp. 5246-5251.

[21] S. V. Rakovic, E. C. Kerrigan, K. I. Kouramas, and D. Q. Mayne, Invariant approximations of the minimal robustly positively invariant sets, IEEE Trans. Automat. Control 50 (2005), no. 3, 406-410.

[22] S. V. Rakovic, E. C. Kerrigan, D. Q. Mayne, and K. I. Kouramas, Optimized robust control invariance for linear discrete-time systems: Theoretical foundations, Automatica J. IFAC 43 (2007), no. 5, 831-841.

[23] S. V. Rakovic, E. C. Kerrigan, D. Q. Mayne, and J. Lygeros, Reachability analysis of discrete-time systems with disturbances, IEEE Trans. Automat. Control 51 (2006), no. 4, 546-561.

[24] R. Vidal, S. Schaffert, O. Shakernia, J. Lygeros, and S. Sastry, Decidable and semidecidable controller synthesis for classes of discrete time hybrid systems, in Proc. 40th IEEE Conf. on Decision and Control, 2001.

Department of Mathematics and Information Sciences

University of North Texas at Dallas

DALLAS, TX 75241, USA

E-mail address: byungik.kahng@unt.edu 